\title{
NUEVAS COMBINACIONES EN MONOCOTILEDÓNEAS MEXICANAS IV (BROMELIACEAE, ORCHIDACEAE)
}

\author{
Ana Rosa López-Ferrari y Adolfo Espejo-Serna \\ Universidad Autónoma Metropolitana-Iztapalapa, \\ División de Ciencias Biológicas y de la Salud, Departamento de Biología, \\ Herbario Metropolitano, Apdo. postal 55-535, 09340 México, D.F. México. \\ aes@xanum.uam.mx
}

\section{RESUMEN}

Se propone elevar a Tillandsia tortilis Klotzsch ex Baker ssp. curvifolia Ehlers et Rauh al nivel específico con el nombre de Viridantha curvifolia (Ehlers et Rauh) LópezFerrari et Espejo. Asimismo se presenta a Malaxis zempoalensis López-Ferrari et Espejo como nuevo nombre para Malaxis palustris Espejo et López-Ferrari.

Palabras clave: Bromeliaceae, Malaxis, México, Tillandsia, Viridantha.

\section{ABSTRACT}

We propose to raise to specific rank to Tillandsia tortilis Klotzsch ex Baker ssp. curvifolia Ehlers et Rauh with the name Viridantha curvifolia (Ehlers et Rauh) LópezFerrari et Espejo. Also, the new name Malaxis zempoalensis López-Ferrari et Espejo is proposed for Malaxis palustris Espejo et López-Ferrari.

Key words: Bromeliaceae, Malaxis, Mexico, Tillandsia, Viridantha.

1. En su revisión del género Viridantha (2002), Espejo-Serna incluyó en el mismo seis especies: $V$. atroviridipetala (Matuda) Espejo, $V$. ignesiae (Mez) Espejo, V. lepidosepala (L. B. Sm.) Espejo, V. mauryana (L. B. Sm.) Espejo, V. plumosa (Baker) Espejo y $V$. tortilis (Klotzsch ex Baker) Espejo y consideró a Tillandsia tortilis Baker ssp. curvifolia Ehlers et Rauh, como sinónimo de $V$. tortilis. La revisión 
de material de dicho género para elaborar el fascículo correspondiente a Bromeliaceae para la Flora del Bajío y de Regiones Adyacentes, así como la recolección de ejemplares en los estados de Hidalgo, Guanajuato y Zacatecas, nos permitió comparar más detalladamente ejemplares, tanto vivos como herborizados, de sendos taxa, pudiendo encontrar diferencias morfológicas significativas entre ambos. Viridantha tortilis incluye plantas más o menos caulescentes con las hojas rectas, generalmente escuarrosas y con espigas aplanadas y elípticas, de 3.5 a $4 \mathrm{~cm}$ de largo, en tanto que las plantas de la supuesta subespecie curvifolia son acaules, con hojas curvadas y nunca escuarrosas y tienen espigas rollizas y elipsoides, de 1.5 a $2 \mathrm{~cm}$ de largo. Todo lo anteriormente dicho nos permite concluir que las plantas descritas como T. tortilis ssp. curvifolia ni son coespecíficas ni representan una variedad de Viridantha tortilis, sino que pertenecen a una especie distinta para la cual proponemos la siguiente combinación:

Viridantha curvifolia (Ehlers et Rauh) López-Ferrari et Espejo, comb. et stat. nov. Basónimo: Tillandsia tortilis Klotzsch ex Baker ssp. curvifolia Ehlers et Rauh, J. Bromeliad Soc. 40: 167-168, f. 7, 8. 1990. Tipo: MÉxıco, Guanajuato, municipio de Guanajuato, apud Guanajuato, 2200-2400 m, 6.III.1985, R. y K. Ehlers EM850201 (holotipo: WU!).

Ejemplares examinados: Guanajuato, municipio de Ocampo, 4-6 km al sur de Santa Bárbara, 2200 m, bosque de Quercus sobre peñas, 29.VI.1996, E. Pérez C. y S. Zamudio R. 3379 (IEB); ca. 4 km al S de Santa Bárbara, 2230-2500 m, ladera de cerro, 7.X.1992, E. Carranza G. y cols. 4332 (IEB); 4 km al S de Santa Bárbara, 2200 $\mathrm{m}$, bosque de encino sobre rocas ígneas, cañada, 30.IV.1996, E. Pérez C. y E. Carranza G. 3330 (IEB); municipio de Guanajuato, Cañada de la Virgen, Agua Sabrosa, $21^{\circ} 10^{\prime} 03^{\prime \prime} \mathrm{N}, 101^{\circ} 12^{\prime} 06^{\prime \prime} \mathrm{W}, 2400$ m, vegetación riparia, 10.VIII.1997, J. MartínezCruz 448 (IEB, MEXU); Querétaro, municipio de Huimilpan, cerro Capula, 1 km al N de la carretera San Pedro - Huimilpan, $20^{\circ} 20^{\prime} \mathrm{N}, 100^{\circ} 18^{\prime} \mathrm{W}, 2650 \mathrm{~m}$, bosque de encino, 14.VIII.1997, L. Hernández S. 4262 (IEB); ZaCATECAS, municipio de Tlaltenango de Sánchez Román, $25 \mathrm{~km}$ después de Corral de Piedra, rumbo a Tlaltenango de Sánchez Román, 2139'41" N, 10309'23" W, 2450 m, lomas con bosque de encino con ericáceas, 19.X. 2007, A. Espejo et al. 7072 (UAMIZ).

Cabe aquí comentar que Ehlers (2004) publicó Tillandsia penascoensis con material procedente del peñón de San Mateo Peñasco, Oaxaca y que Espejo y López-Ferrari (Espejo-Serna et al., 2007, p. 140) publicaron la combinación de este 
epíteto en Viridantha, género que queda entonces conformado por ocho especies, dos más de las citadas para México por Espejo-Serna et al. (2004), todas endémicas de México.

Todos los taxa del género Viridantha constituyen un grupo bien delimitado que se distingue claramente de Tillandsia sensu stricto por el siguiente conjunto de caracteres (Espejo, 2002): flores proterandras, dísticas, descendentes, con los pétalos acintados de color verde oscuro, de 2 a $3 \mathrm{~cm}$ de largo, la corola no constreñida en la base para formar una garganta, estambres inclusos, todos iguales en longitud, con los filamentos filiformes y aplanados, anteras semibasifijas y estigma tipo simpleerecto. Las rosetas en Viridantha son de tipo compacto, no forman tanque y tienden a la caulescencia, además de presentar escamas largamente asimétricas, densa a muy densamente dispuestas (Espejo, 2002).

2. Cuando publicamos el nombre de Malaxis palustris Espejo et López-Ferrari (Espejo-Serna et al., 1998), inadvertidamente pasamos por alto que dicho binomio había sido ya aplicado por Richard (1818) a otra especie del mismo género, por lo cual se hace necesario el siguiente nombre nuevo:

Malaxis zempoalensis López-Ferrari et Espejo, nomen novum. Basónimo: Malaxis palustris Espejo et López-Ferrari, Sida 18: 412. 1998, non Malaxis palustris Rich., 1818. Tipo: México, Morelos, municipio de Huitzilac, $1-2 \mathrm{~km}$ al W de la laguna Zempoala, sobre el arroyo Las Trancas, parque nacional Lagunas de Zempoala, $19^{\circ} 02^{\prime} \mathrm{N}$, 99¹9’ W, 2800 m, 12.VII.1997, A. Espejo, A. R. López-Ferrari, J. García-Cruz y R. Jiménez M. 5714 (holotipo: UAMIZ; isotipos: AMO, AMES).

Ejemplares examinados: Morelos, municipio de Huitzilac, $1-2 \mathrm{~km}$ al $\mathrm{W}$ de la laguna Zempoala, sobre el arroyo Las Trancas, parque nacional Lagunas de Zempoala, 1902'37" N, 99¹9'20" W, 2800 m, 12.VII.1997, A. Espejo et al. 5714 (AMES, AMO, UAMIZ); $1-2 \mathrm{~km}$ al W de la laguna Zempoala, sobre el arroyo Las Trancas, parque nacional Lagunas de Zempoala, 1902'37", 99¹9'20" W, 2800 m, 12.VII.1997, J. García-Cruz et al. 751 (AMO, UAMIZ).

\section{AGRADECIMIENTOS}

Deseamos expresar nuestro agradecimiento a Jerzy Rzedowski y Graciela Calderón por la revisión y las atinadas sugerencias al manuscrito. Los curadores de 
los siguientes herbarios AMO, IEB, MEXU, UAMIZ y WU nos brindaron todas las facilidades para consultar sus colecciones.

\section{LITERATURA CITADA}

Ehlers, R. 2004. Tillandsia penascoensis Ehlers \& Lautner spec. nov. Bromelie 2004(1): 4-7.

Espejo-Serna, A., A. R. López-Ferrari, J. García-Cruz, R. Jiménez M. y L. Sánchez A. 1998. Dos nuevas adiciones a la orquideoflora mexicana. Sida 18(2): 411-418.

Espejo-Serna, A. 2002. Viridantha, un género nuevo de Bromeliaceae (Tillandsioideae) endémico de México. Acta Bot. Mex. 60: 25-35.

Espejo-Serna, A., A. R. López-Ferrari, I. Ramírez-Morillo, B. K. Holst, H. E. Luther \& W. Till. 2004. Checklist of Mexican Bromeliaceae with notes on species distribution and levels of endemism. Selbyana 25: 33-86.

Espejo-Serna, A., A. R. López-Ferrari, N. Martínez-Correa y V. A. Pulido-Esparza. 2007. Bromeliad flora of Oaxaca, Mexico: richness and distribution. Acta Bot. Mex. 81: 71-147.

Richard, L. 1818. De Orchideis Europaeis annotationes. Mem. Mus. Hist. Nat. 4: 23-61. 